\title{
Achieving Better Greenhouse Effect than Glass: Visibly Transparent and Low Emissivity Metal-Polymer Hybrid Metamaterials
}

\author{
Tao $\mathrm{Li}^{1,2, t}$, Yin Gao ${ }^{1, \hbar}$, Kun Zheng ${ }^{3, \dagger}$, Yongmei Ma ${ }^{3}$, Ding Ding ${ }^{4, *}$ and Hang Zhang ${ }^{1,2, *}$
}

Common glass is absorbing in the mid-infrared but transparent to sunlight, keeping our greenhouses and rooms warm. But a visibly-transparent and infrared-reflective material will perform much better than glass. Engineered multilayer optical coatings have been able to achieve both visible transparency and infrared reflectivity, but manufacturing cost has prevented their use on a large scale. Here, we predicted and successfully synthesized a transparent wavelength-selective metal-polymer hybrid films with low emissivity of less than 0.1 in the infrared range. The films, based on silver nanowires and PMMA, exhibit high transmission ( $>85 \%)$ through the visible wavelength range and high reflectance $(>90 \%)$ in the mid-wavelength and long-wavelength infrared range. Our films are more transparent than a commercially available multilayer engineered coating in the visible and are much easier to fabricate. On an average sunny day, our films in this work warm up a prototype greenhouse 8 degrees Celsius higher than that of glass. We believe that our films hold promise for large scale applications, leading to significant energy savings for indoor heating.

Keywords: Geenhouse effect; Low emissivity; Metamaterial; Radiation regulation

Received 21 August 2019, Accepted 23 August 2019

DOI: $10.30919 /$ esee 8 c325

\section{Introduction}

A growing population means greater energy demand. We consume a significant amount of energy on maintaining thermal comfort ${ }^{1}$ and it is no surprise that reducing heat loss is one of the most important methods to reduce energy consumption and improve energy efficiency. ${ }^{2}$ For instance, $25 \%-35 \%$ of energy wasted in buildings is due to inefficient windows ${ }^{3}$ and using superior double glazed windows can save us 10 billion dollars annually. ${ }^{4}$ Thus, energy efficient windows have been on high demand. Currently, there are two main categories of the energy efficient windows available on the market. One is fluorinated tin dioxide films deposited at high temperatures by pyrolytic chemical vapor deposition; ${ }^{5}$ the other is a thin layer of metal surrounded by dielectric protective layers by magnetron sputtering. ${ }^{6}$ More recently, a metal-based multilayer coating and doped semiconductor with a wide band gap such as doped indium tin oxides (ITO) ${ }^{8}$ and aluminium-doped zinc oxide $(\mathrm{AZO})^{9}$ have been used to develop energy efficient windows.

Fig. 1 shows the concept of how a greenhouse warms us up.

${ }^{1}$ Institute of Engineering Thermophysics, Chinese Academy of Sciences, Beijing, China

${ }^{2}$ University of Chinese Academy of Sciences, Beijing, China

${ }^{3}$ Institute of Chemistry, Chinese Academy of Sciences, Beijing, China

${ }^{4}$ Institute of Materials Research and Engineering, A*STAR Research Entities, Singapore

*E-mail:ding_ding@imre.a-star.edu.sg; zhanghang@iet.cn

$\uparrow$ Author Contributions

Tao Li, Yin Gao and Kun Zheng contributed equally and should be considered co-first authors
Normal glass allows sunlight to heat up objects and absorbs the thermal radiation emitted by them, then re-emitting thermal radiation inwards and outwards at the same time, creating a greenhouse. A perfect lowemissivity (Low-E) window exhibits high transmittance throughout the visible spectral region and high reflectance in the infrared region. Hence, no thermal radiation is re-emitted outwards. ${ }^{10}$ In addition to the energy-saving windows in buildings, ${ }^{11}$ Low-E coatings can be widely used in aerospace to reduce the absorption of thermal radiation, ${ }^{12,13}$ textile, ${ }^{14}$ and automobile windows. ${ }^{15}$

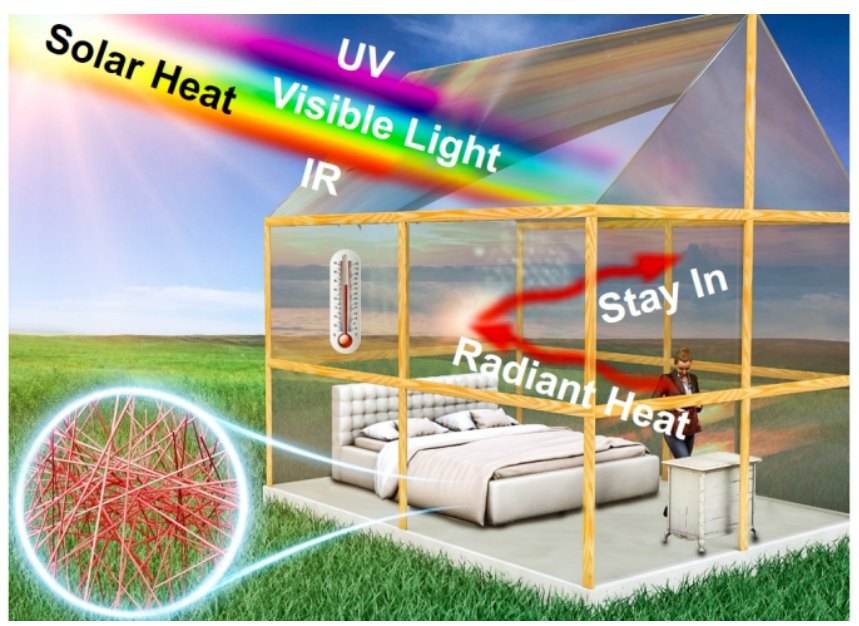

Fig. 1 Window with Low-E coating for heat conduction showing the concept of how a greenhouse warms us up. The Low-E coating helps to retain almost all the emitted infrared radiation and helps to enhance the greenhouse effect unlike glass which still re-emits a significant portion of the radiation outwards. 
Recently, the low cost of polymers and the ease to process polymers on large scale processing have led to promising applications in the production optical components. ${ }^{16-18}$ Meanwhile, silver nanowires (AgNWs) films are actually transparent in the visible, as evident in applications such as touch screens, organic solar cells and flat panel display, ${ }^{19,20}$ and offer a lower cost compared to ITO or AZO. ${ }^{21}$ Furthermore, AgNWs have been used in heat retaining fabrics. ${ }^{14,22}$ Here, we carried polymeric photonics with AgNWs and develop a polymeric metamaterial that warms our greenhouses better. Films in this work have much simpler fabrication and potentially much lower cost compared to current Low-E technology. Moreover, our metamaterials show compatible, and if not superior, performance compared to current Low-E materials and demonstrates actual improvement under solar heating compared to glass.

\section{Experimental}

\subsection{Film preparation}

We used a method from Korte et al. ${ }^{23}$ to make the AgNWs. Here, several types of AgNWs with the average diameters of $20 \mathrm{~nm}, 50 \mathrm{~nm}$, $70 \mathrm{~nm}$ and $90 \mathrm{~nm}$ and the length of about $20 \mu \mathrm{m}$ which were dispersed in with the concentration of $2 \mathrm{mg} / \mathrm{ml}$ were synthesized through the salt mediated polyol process. Then, $100 \mathrm{~mL}$ of Glycol (EG) and 0.05-0.08 $\mathrm{mM}$ of $\mathrm{NaCl}$ were added into a disposable glass vial and heated in an oil bath with the temperature of $185{ }^{\circ} \mathrm{C}$ for $1 \mathrm{~h}$ under magnetic stirring at $250 \mathrm{rpm}$. After mixing, $0.01-0.02 \mathrm{mM}$ of $\mathrm{CuCl}_{2}$ solution was added into the heated $\mathrm{EG}$ and heated for $20 \mathrm{~min}$. In the last step, $10 \mathrm{~mL}$ of 0.19 M Polyvinyl pyrrolidone (PVP) solution in EG was added into the solution followed by $10 \mathrm{~mL}$ of a $0.001-0.004 \mathrm{mM} \mathrm{AgNO}$ solution. After AgNWs were formed, the vial was quenched in water bath at room temperature. Then products were washed in acetone and isopropanol to remove impurities.

The detailed experiment process is shown in Fig. 2. To mix the AgNWs in the polymer matrix, we used PMMA and anisole with purity of $\geq 99.5 \%$ which were supplied from Aladdin, China. PMMA was dissolved in anisole with magnetic stirring for $24 \mathrm{~h}$ to get PMMAanisole solution with PMMA concentration of 4 wt.\%. Then AgNWsisopropanol solution and PMMA-anisole solution with volume ratio of 1:2 were mixed by magnetic stirring for $1 \mathrm{~h}$. Various methods such as spin-coating, dip-coating, spray-coating, slit-coating etc. could be used for the preparation of these metal-polymer hybrid metamaterial. ${ }^{24-26} \mathrm{In}$ this work, AgNWs-PMMA films were prepared by dipping the substrates in the prepared sol using a dip-coater (model SYDC-100H, Shanghai Sanyan Technology Co., Ltd.) at room temperature as shown in Fig. 2. The substrates are immersed into the solution at a constant speed of $1000 \mu \mathrm{m} / \mathrm{s}$ followed by a dwell time of $20 \mathrm{~s}$, and then pulled out of the solution at a constant speed of $1000 \mu \mathrm{m} / \mathrm{s}$ and dried under ambient conditions for $30 \mathrm{~s}$ during which the solvent was evaporated and thin films were formed on the substrates. This process was repeated for 10, 20, and 30 times to get different thickness of AgNWs. After dipcoating, the samples were dried at temperature of $50{ }^{\circ} \mathrm{C}$ and pressure of $10^{-1} \mathrm{~Pa}$ for $2 \mathrm{~h}$.

We have used different substrates to hold our AgNWs metamaterials for different measurements. Glass substrates with the thickness of $3 \mathrm{~mm}$ and single crystal $\mathrm{KBr}$ substrates with the thickness of $3 \mathrm{~mm}$ were supplied from Schott (Germany) and Sunson (China), respectively. Si substrates were used for the infrared spectra measurements in order to characterize the emissivity of the films. All substrates were cleaned with acetone and isopropanol successively each for $15 \mathrm{~min}$ in ultrasonic bath.

\subsection{Characterization}

The film thickness was determined by a surface profiler (Bruker Nano Dektak XT). A diamond stylus with a radius of $5 \mu \mathrm{m}$ was moved with a normal force of $9.8 \times 10^{-3} \mathrm{mN}$ on the sample surface during the measurement. Each profile was measured at a scan speed of $200 \mu \mathrm{m} / \mathrm{s}$ and scan length of $4 \mathrm{~mm}$ and a frequency of $200 \mathrm{~Hz}$. The Infrared spectra were measured with a Fourier transform infrared (FTIR) spectrometer (Spectrum One, Perkin Elmer Instruments Co. Ltd., USA) in the range of $400-4000 \mathrm{~cm}^{-1}$ in plane reflection modes. An incident angle of $25^{\circ}$ with respect to the sample surface normal and a resolution of $2 \mathrm{~cm}^{-1}$ were applied during the measurements. The ultraviolet-visiblenear infrared (UV-VIS-NIR) spectra were recorded in the wavelength range of $300 \mathrm{~nm}<\lambda<1400 \mathrm{~nm}$ using an UV-VIS-NIR SpectroPhotometer (Lambda-950), and measured in the transmission modes. Pure substrates were used as references for the characterization of coated samples. The surface morphology was characterized using a scanning electron microscope (SEM) (Zeiss, Merlin Compact) at an operation voltage of $10 \mathrm{kV}$. The infrared emissivity value $(\varepsilon)$ of the films on the single crystal $\mathrm{KBr}$ were calculated from the infrared transmittance and reflectance values by Eq. (1) and (2). ${ }^{27}$ The infrared radiation properties of the films are characterized with spectral range of $7.5-14 \mu \mathrm{m}$ at $50{ }^{\circ} \mathrm{C}$ by infrared thermographic camera (InfraTec
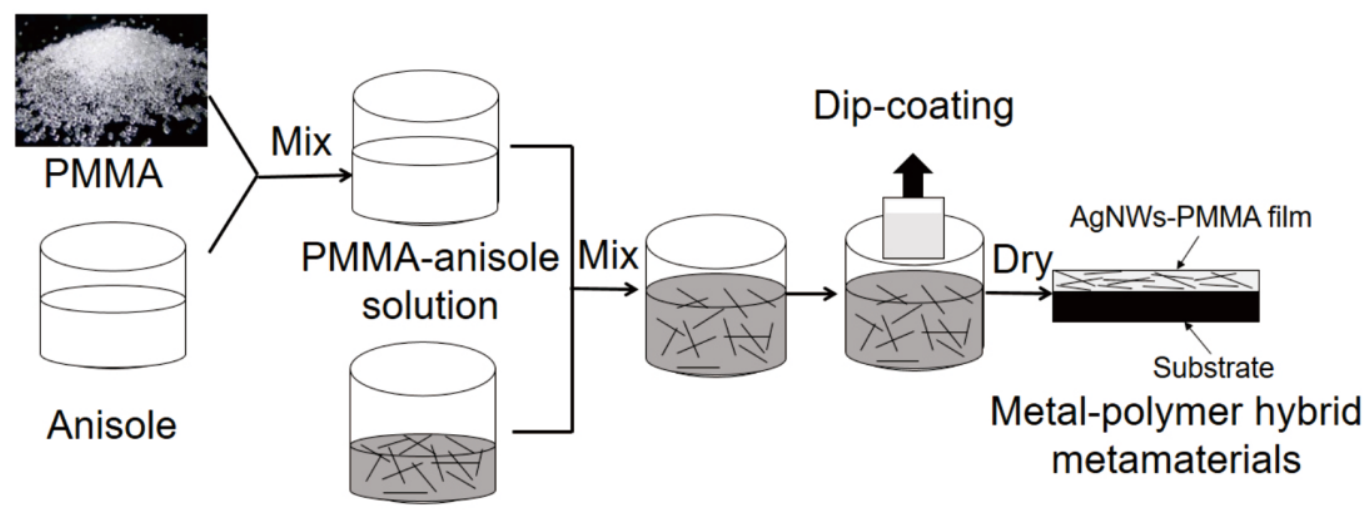

\section{AgNWs-isopropanol solution}

Fig. 2 Materials and preparation process. 
$\mathrm{GmbH})$. Further, in order to confirm the effective radiative heating of the AgNWs-PMMA film, a comparable experiment was carried on. Two small houses with glass windows (ones were uncoated glasses and the others were glasses coated by AgNWs-PMMA film, which are shown in Fig. 6 (a) and (b)) were put under the sun for 3 hours and the temperatures in the small houses were recorded. Furthermore, the optical properties of AgNWs-PMMA films and the commercial double Low-E glasses supplied from TAIWAN GLASS IND. CORP are compared.

\section{Theory and simulation}

Emissivity $(\varepsilon)$ of a material is how close it resembles that of a blackbody. A Low-E material will have a low value of $\varepsilon$, implying that the material emits much lesser than a blackbody. From conservation of energy, $\varepsilon+\rho+\tau=1$ (1) and $\varepsilon=\alpha$ (2) where $\varepsilon, \rho, \tau$ and $\alpha$ are emissivity, reflectivity, transmissivity and absorptivity, respectively. Thus, decreasing the emissivity value implies increasing its reflectivity.

To understand how our AgNWs metamaterials perform in the visible and infrared, we have to be able to translate how each AgNW scatter into the reflectance of the entire film. AgNWs exhibit plasmonic effects in the visible ${ }^{21,28,29}$ and its scattering is strongly influenced by the metal, the shape and the size. ${ }^{29,30}$ The transparency of AgNWs films in the visible regime have been reported before. ${ }^{21,32,3,33}$ Here, we use Mie scattering formulation in order to obtain the effective index of refraction. ${ }^{34,35,36}$ The effective index is then used to calculate the transmission and reflection coefficients using the transfer matrix method. ${ }^{37}$ In this calculation, we assume that the volume fraction of the $\mathrm{AgNW}$ relative to the polymer is low (less than $10 \%)^{38}$ for such methods to be accurate.

\section{Results and discussion}

AgNWs-PMMA films with an average thickness of around $300 \mathrm{~nm}$ in which the diameter of AgNWs is $20 \mathrm{~nm}$ were synthetized. The film shows completely homogeneous in both macroscopic and microscopic regime which are shown in Fig. 3. A transparent film on the glass is visible as shown in Fig. 3 (a). Fig. 3 (b) shows the SEM image obtained for the AgNWs-PMMA film on the glass substrate. Homogeneous nanowire networks and AgNWs with the diameter of $20 \mathrm{~nm}$ which are uniformly distributed in PMMA matrix are observed. The measured and the calculated transmittance spectrum of the AgNWs-PMMA hybrid
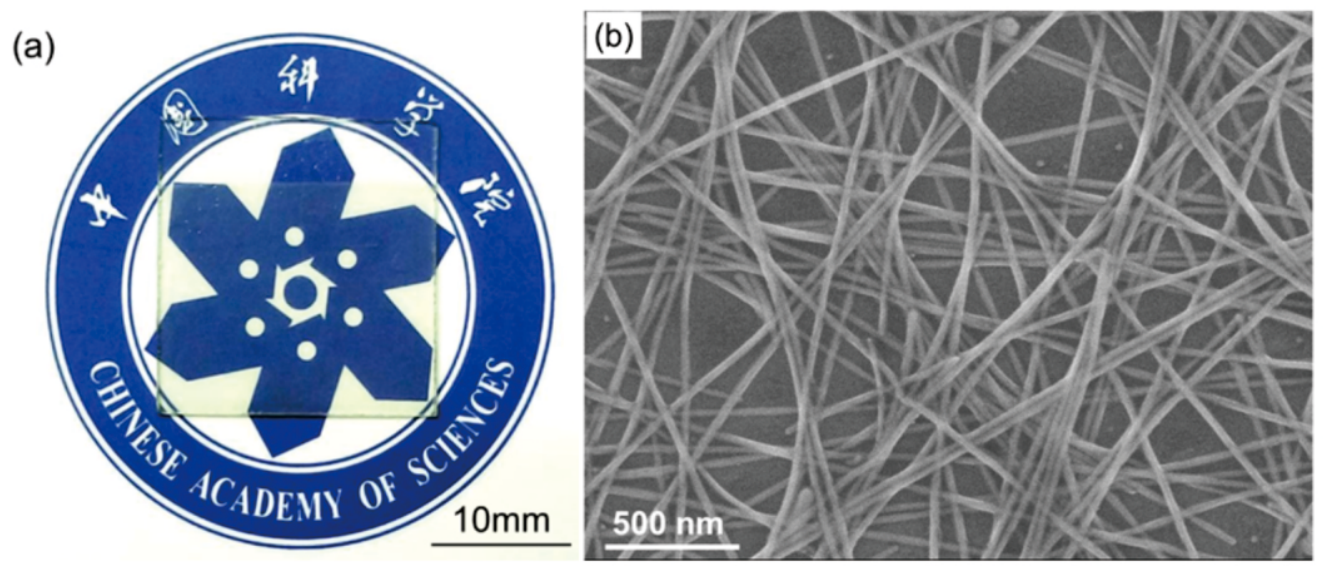

Fig. 3 Macroscopic view and microstructure of the metal-polymer hybrid meta-material. The (a) optical and (b) SEM image of a metal-polymer hybrid film deposited on a glass substrate.

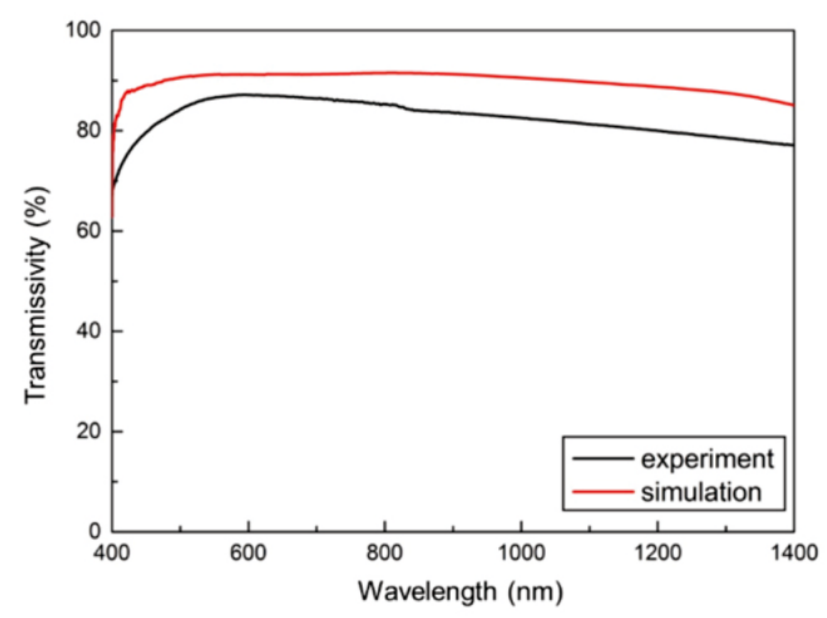

Fig. 4 The measured (black curve) and the calculated (the red curve) spectral transmittance in the visible light and near infrared wavelength range for the AgNWs-PMMA films deposited on the glass. The calculation is based on the effective medium theory.

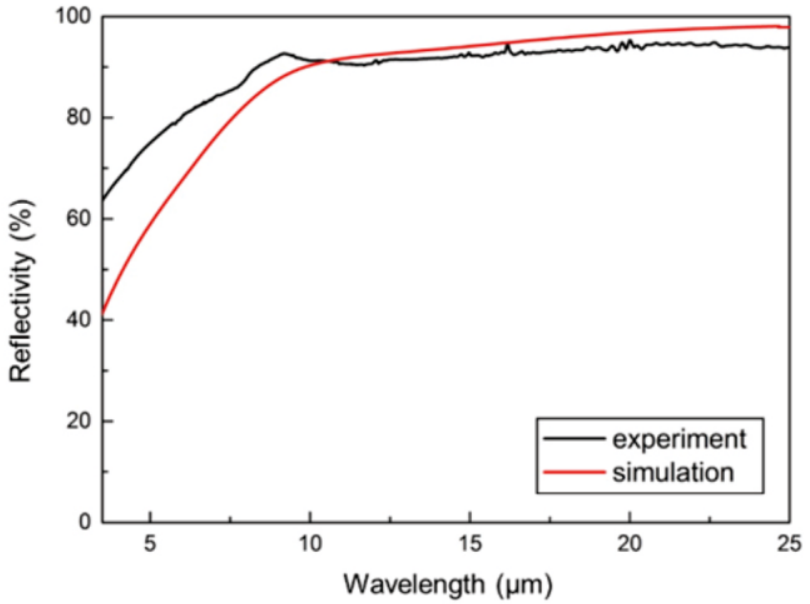

Fig. 5 The measured (black curve) and the calculated (the red curve) spectral reflectance in the mid-wavelength and long-wavelength infrared for the AgNWs-PMMA film deposited on the glass. The calculation is based on the effective medium theory. 


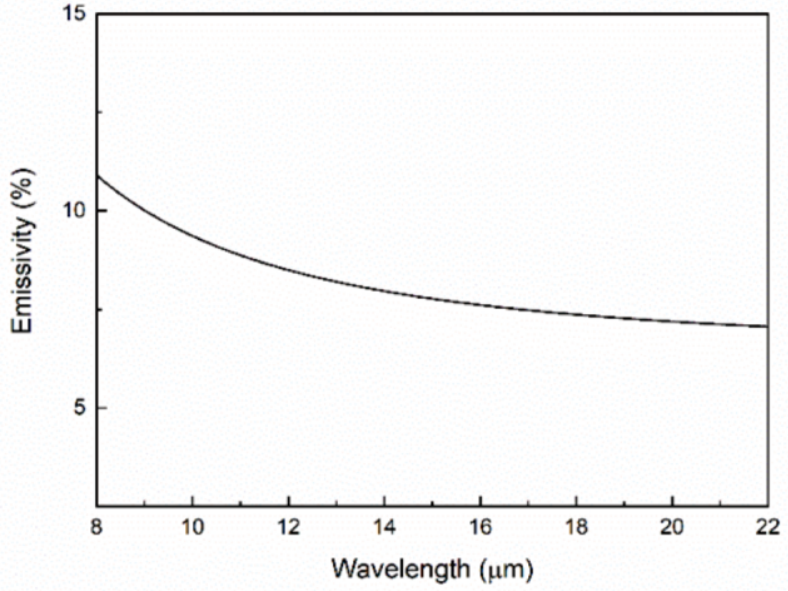

Fig. 6 Infrared emissivity of the AgNWs-PMMA film with thickness of around $300 \mathrm{~nm}$ deposited on the $\mathrm{KBr}$ substrate. film on the glass in visible light and near infrared wavelength range were determined as shown in Fig. 4. The film possesses a stable transmittance around $85 \%$ in the visible light range, which means the most visible light can transmit through this film. This is very important for the energy-saving windows. The difference of the transmissivity between the experiment and the simulation is less than $5 \%$. Considering PMMA exhibits high transmission through the visible wavelength range (around $90 \%$ ), ${ }^{39,40}$ around $85 \%$ of the visible light which can transmit through the AgNWs-PMMA film is estimated, which is in agreement with the measured result as shown in Fig. 4. Fig. 5 shows the spectral reflectance of the film in the infrared region using FTIR. More than $90 \%$ of the infrared light (especially the long-wavelength infrared) could be reflected by the AgNWs-PMMA hybrid film. The infrared emissivity (absorptivity) of the films on the single crystal $\mathrm{KBr}$ is less than 0.1 as shown in Fig. 6, which is in agreement with the reflectivity value of $>90 \%$. Furthermore, the effective radiative heating of the AgNWs-PMMA film is confirmed by comparable experiments in prototype greenhouses and the results are shown in Fig. 7. The temperature in the house with glass windows coated by metamaterial is higher than the house with uncoated glass windows throughout all the time during the continuous experiment and rises more rapidly. The maximum difference in temperature between these two houses reaches up to $8{ }^{\circ} \mathrm{C}$.

Furthermore, the optical performance of the AgNWs-PMMA
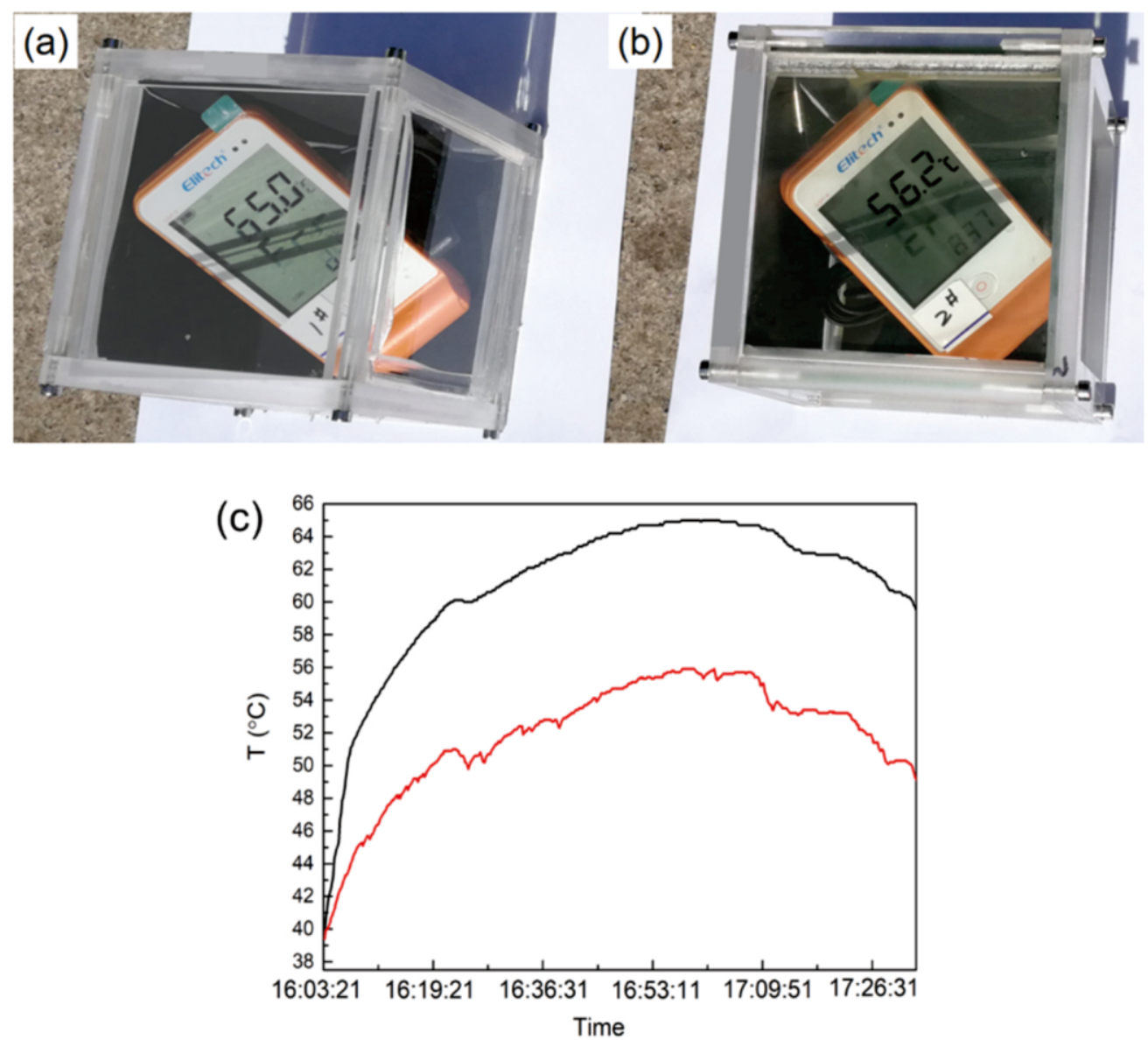

Fig. 7 Performance of the AgNWs-PMMA films for effective radiative heating. (a) and (b) show photos indicating the temperatures in the prototype greenhouses with and without AgNWs-PMMA films, respectively. (c) Dependence of the temperature in the prototype greenhouses on the time. The black and red curves show the temperature of the simulated houses with the windows coated with and without AgNWs-PMMA films, respectively. 
films with film thickness of $300 \mathrm{~nm}$ and the AgNWs diameter of $20 \mathrm{~nm}$ and the commercial Low-E glasses is shown in Fig. 8. The visible light transmissivity of the AgNWs-PMMA film is around $85 \%$, which is larger than the value of commercial double Low-E glass (only 40\%), due to the Mie scattering and surface plasmon enhanced transmission. The reflectivity of the AgNWs-PMMA film in the wavelength of 9 - 22 $\mu \mathrm{m}$ where the infrared range under room temperature is $95 \%$, which is similar to the commercial double Low-E glass.

Finally, the optical property of the AgNWs-PMMA films with the same thickness of around $500 \mathrm{~nm}$ consisting AgNWs with different diameters of $20 \mathrm{~nm}, 50 \mathrm{~nm}, 70 \mathrm{~nm}$ and $90 \mathrm{~nm}$ are investigated as shown in Fig. 9 (a). With decreasing the diameter of AgNWs, the reflectivity in the infrared range increases, which indicates the smaller diameter of metal nanowire in metal-polymer hybrid metamaterials is the better energy saving effect it yielda. Fig. 9 (b) shows the reflectivity of the AgNWs-PMMA films with the same thickness of around $500 \mathrm{~nm}$ consisting with different diameters of AgNWs in the infrared which was calculated by the effective medium theory. From Fig. 9, we can observe similar behaviors of reflection in infrared range between the experimental and the theoretical results have results have shown. The thinner the nanowires are the more it appears to be a dipole at longer wavelengths, exhibiting Rayleigh scattering which is more backward scattering than at higher orders.

\section{Conclusions}

Transparent wavelength-selective metal-polymer hybrid metamaterials with low emissivity of less than 0.1 in the infrared range were prepared by sol-gel process which is an economical method for large scale applications. The metamaterials based on AgNWs and PMMA show excellent optical and energy efficient performance with high transmission of more than $85 \%$ through the visible wavelength range and high reflectance of more than $90 \%$ in the mid-wavelength and long- (a)

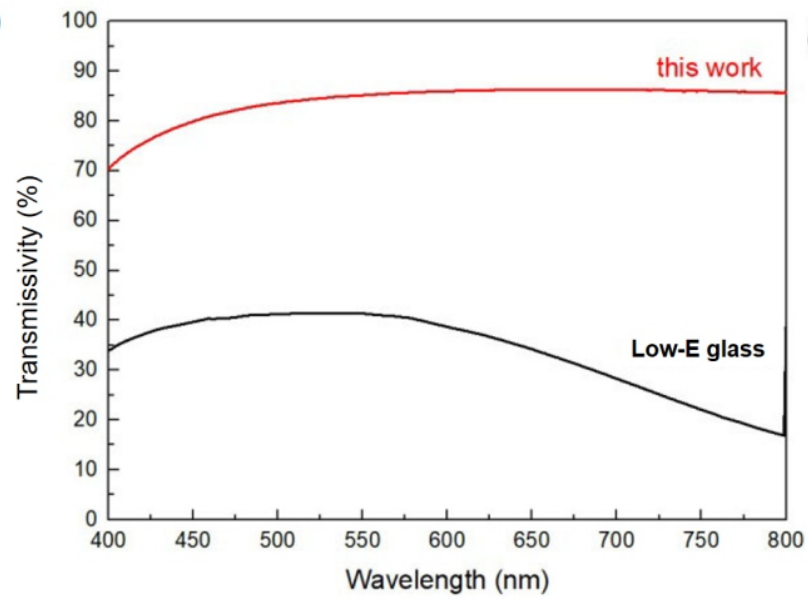

(b)

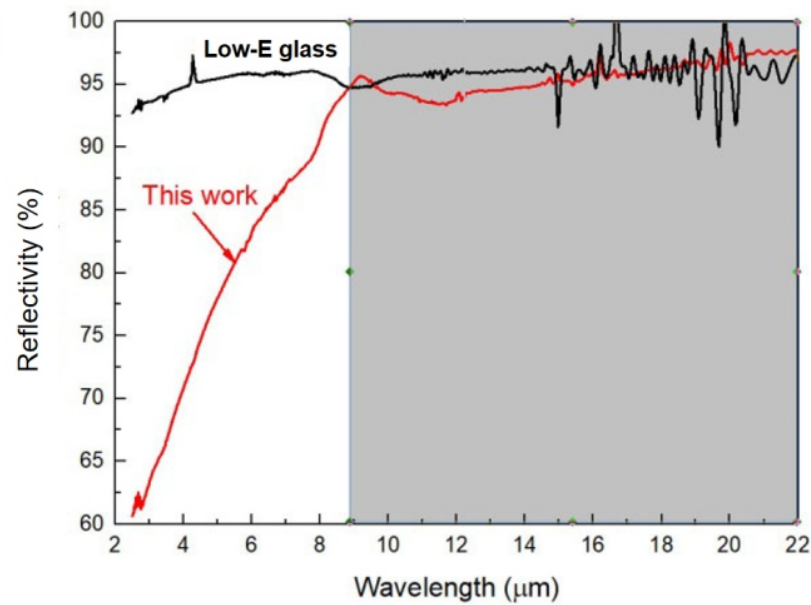

Fig. 8 Visible light and infrared spectrum properties of the AgNWs-PMMA films and commercial Low-E glasses.

(a)

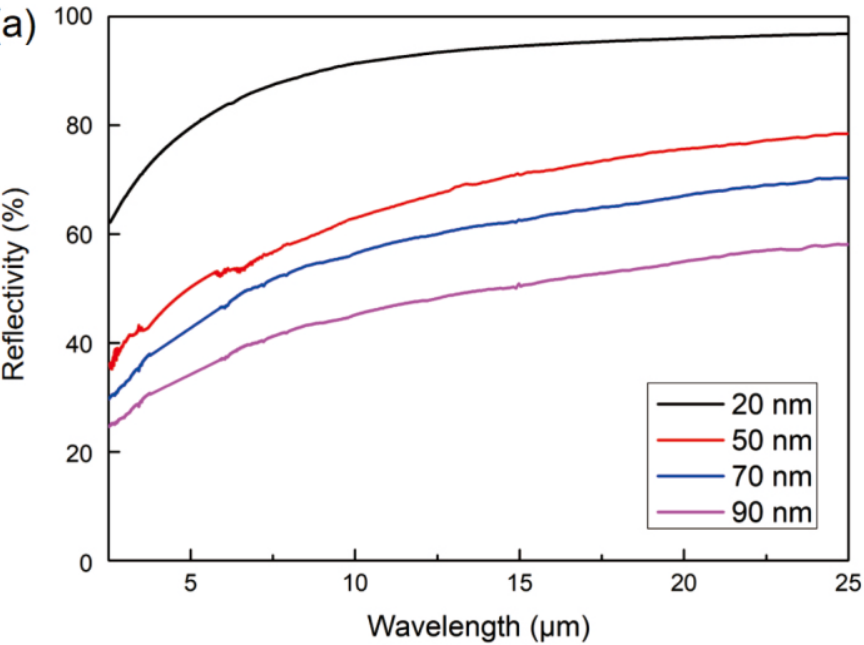

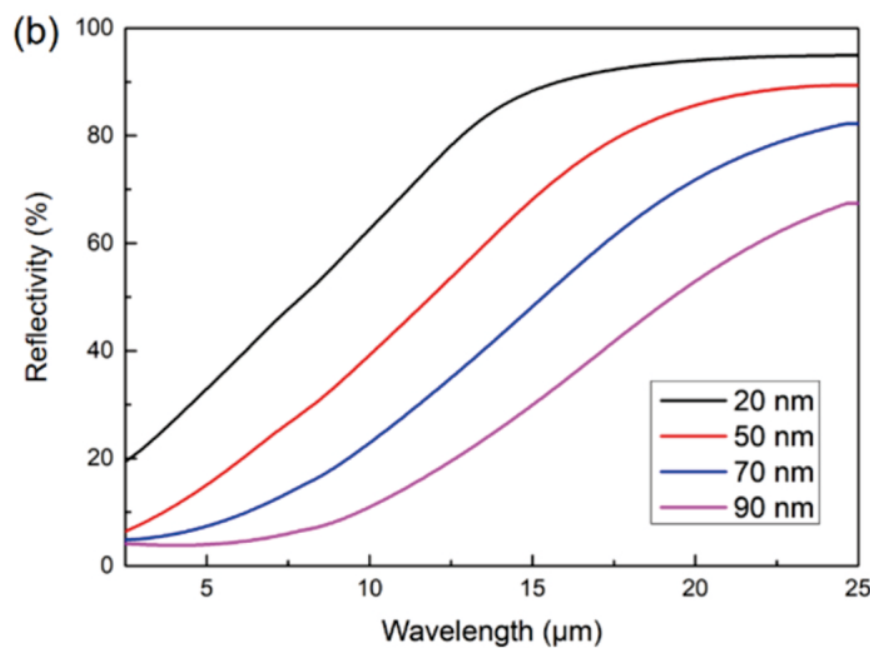

Fig. 9 (a) The measured spectral reflectance in the mid-wavelength and long-wavelength infrared for the PMMA film and AgNWs-PMMA films consisting of AgNWs with different diameters. (b) The calculated spectral reflectance for AgNWs-PMMA films consisting of AgNWs with different diameters which is based on the effective medium theory. The black, red, green, magenta and blue curves indicate the spectral reflectance property for the pure PMMA film, the AgNWs-PMMA film with the AgNWs diameter of $20 \mathrm{~nm}, 50 \mathrm{~nm}, 70 \mathrm{~nm}$ and $90 \mathrm{~nm}$, respectively. 
wavelength infrared range. The performance is better than commercial double Low-E glasses. Theoretical calculation was carried out to show that AgNWs exhibit plasmonic effects in the visible light range and is influenced by the metal and the geometric parameters, which is in an agreement with the experimental results. In comparison to the prototype greenhouses with uncoated glass windows, the house with glass windows coated by metamaterial could warm up 8 degrees Celsius more. Furthermore, the optical properties of the AgNWs-PMMA films with different diameters were investigated. The reflectivity in the infrared range increases with decreasing diameter of AgNWs. Overall, our film holds great promise for providing a cost-effective method to achieve state-of-the-art Low-E coatings for greater energy saving.

\section{Conflict of interest}

There are no conflicts to declare.

\section{Acknowledgements}

This work is supported by the Basic Science Center Program for Ordered Energy Conversion of the National Natural Science Foundation of China (No.51888103) and the CAS Pioneer Hundred Talents Program. D. D. acknowledges support by AME Young Individual Research Grants by the Agency for Science, Technology and Research under Grant No. A1884c0020. We would like to thank Jaron Lim for contributing to the optical calculations in this work.

\section{Competing interests}

Patents (CN 108995103 A and CN 109181455 A) have been granted related to this work.

\section{References}

1. S. Barbhuiya and S. Barbhuiya, Build. Environ., 2013, 68, 1-11.

2. E. Cuce, C. H. Young and S. B. Riffat, Energy Build., 2015, 86, 595-600.

3. M. Casini, Proc. of the Second Intl. Conf. on Advances in Civil, Structural and Environmental Engineering - ACSEE, 2014, 273-281.

4. Julie Chao, 'Super Window' Could Save \$10 Billion Annually in Energy Costs. Berkeley Lab 2018, 510, 486-6491.

5. D. S. Bhachu, M. R. Waugh, K. Zeissler, W. R. Branford and I. P. Parkin, Chem. - A Eur. J., 2011, 17, 11613.

6. Hill and Russ, Coated Glass Applications and Markets, BOC Coating Technology, Fairfield, CA, 1999.

7. R. J. Martin-Palma, L. Vázquez, J. M. Martinez-Duart and MalatsRiera, Sol. Energ. Mat. Sol. C, 1998, 53, 760-778.

8. C. G. Granqvist and A. Hultåker, Thin Solid Films, 2002, 411, 1-5.

9. D. Zhu, K. Li, F. Luo and W. Zhou, Appl. Surf. Sci., 2009, 255, 6145-6148.

10. C. Schaefer, G. Bräuer and J. Szczyrbowski, Surf. Coatings Technol., 1997, 93, 37-45.
11. E. Hammarberg and A. Roos, Thin Solid Films, 2003, 442, 222-226.

12. K. Sun, W. Zhou, X. Tang and F. Luo, Infrared Phys. Technol., 2016, 78, 156-161.

13. Z. Mao, W. Wang, Y. Liu, L. Zhang, H. Xu and Y. Zhong, Thin Solid Films, 2014, 558, 208-214.

14. P. C. Hsu, X. Liu, C. Liu, X. Xie, H. R. Lee, A. J. Welch, T. Zhao and Y. Cui, Nano Lett., 2015, 15, 365-371.

15. Eric Eby, Roger O'Shaughnessy and Robert Bond, High Transmittance, Low Emissivity Coating for Substrates, 1994, 5,302-449.

16. Y. Zhai, Y. Ma, S. N. David, D. Zhao, R. Lou, G. Tan, R. Yang and X. Yin, Science, 2017, 355, 1062-1065.

17. P. N. Prasad, Int. J. Nonlinear Opt. Phys. 1994, 3, 531-541.

18. S. D. Hart, G. R. Maskaly, B. Temelkuran, P. H. Prideaux, J. D. Joannopoulos and Y. Fink, Science, 2002, 296, 510-513.

19. K. Naito, N. Yoshinaga, E. Tsutsumi and Y. Akasaka, Synth. Met., 2013, 175, $42-46$.

20. S. Hemmati, D. P. Barkey, N. Gupta and R. Banfield, ECS J. Solid State Sci. Technol., 2015, 4, 3075-3079.

21. R. Jarrett, H. Kanda, N. Harano, T. Noguchi and R. Crook, Sol. Energy, 2015, 116, 257.

22. C.Y. Chen, M. X. Jing, Z. C. Pi, S. W. Zhu and X. Q. Shen, Nanoscale Res Lett., 2015, 10, 1-7.

23. K. E. Korte, S. E. Skrabalak and Y. Xia, J. Mater. Chem., 2008, 18, 437-441.

24. L. Yan, M. Shao, H. Wang, D. Dudis, A. Urbas and B. Hu, Adv. Mater, 2011, 23, 4120-4124.

25. C. Massard, L. Bernard, R. Cueff, V. Raspal, E. Feschet-Chassot, Y. Sibaud, V. Sautou and K. O. Awitor, Prog. Org. Coatings, 2012, 75, 116-123.

26. H. Youn, K. Jeon, S. Shin and M. Yang, Org. Electron. physics, Mater. Appl., 2012, 13, 1470-1474.

27. P. Pigeat, D. Rouxel and B. Weber, Phys. Rev. B - Condens. Matter Mater. Phys., 1998, 57, 9293-9300.

28. S. Underwood and P. Mulvaney, Langmuir, 1994, 10, 3427-3430.

29. U. Y. Qazi and R. Javaid, Adv. Nanoparticles, 2016, 5, 27-43.

30. T. Tani, S. Hakuta, N. Kiyoto and M. Naya, Opt. Express, 2014, 22, 9262.

31. M. Bobinger, D. Angeli, S. Colasanti, P. La Torraca and L. Larcher, P. Lugli, Phys. Status Solidi A, 2016, 214, 1-11.

32. G. Khanarian, J. Joo, X. Q. Liu, P. Eastman, D. Werner, K. O'Connell and P. Trefonas, J. Appl. Phys., 2013, 114, 024302.

33. M. C. Larciprete, A. Albertoni, A. Belardini, G. Leahu, R. Li Voti, F. Mura, C. Sibilia, I. Nefedov, I. V. Anoshkin, E. I. Kauppinen and A. G. Nasibulin, J. Appl. Phys., 2012, 112, 083503.

34. Hendrik Christoffel Hulst H. C. van de Hulst, Light Scattering by Small Particles, New York: Dover Publications, 1981

35. G. Grzela, D. Hourlier and J. Gómez Rivas, Phys. Rev. B - Condens. Matter Mater. Phys., 2012, 86, 045305.

36. M. S. Wheeler, J. S. Aitchison, J. I. L. Chen, G. A. Ozin and M. Mojahedi, Phys. Rev. B - Condens. Matter Mater. Phys., 2009, 79, 073103.

37. C. C. Katsidis and D. I. Siapkas, Appl. Opt., 2002, 41, 3978.

38. S. J. Byrnes, arXiv:1603.02720 [physics] n.d.

39. E. Shobhana, Int. J. Mod. Eng. Res., 2012, 2, 1092.

40. R. M. Ahmed, Int. J. Photoenergy, 2009, 150389.

Publisher's Note Engineered Science Publisher remains neutral with regard to jurisdictional claims in published maps and institutional affiliations. 